\title{
Habilidades metacomprensivas en estudiantes de profesorado: la formulación de preguntas
}

\author{
Metacomprehension Abilities in Students of Teaching: \\ Question Making
}

\author{
Elvira Arnoux \\ Sylvia Nogueira \\ Adriana Silvestri*
}

Resumen

Ante las crisis de la institución escolar y las propuestas recurrentes de reformas de leyes educativas, resulta imprescindible la elaboración de diagnósticos sobre problemas de alumnos y docentes, entre otras cuestiones. Para hacer un aporte en este sentido, un equipo de investigación dirigido por la profesora Elvira Arnoux, en el Instituto de Lingüística de la Universidad de Buenos Aires, ha diseñado y administrado una encuesta sobre lectura y una prueba de comprensión y metacomprensión para un nivel de educación superior que ha sido objeto de escasos trabajos de investigación: los alumnos de institutos de formación docente para los niveles inicial, primario y secundario de la provincia de Buenos Aires (Argentina). En el presente artículo se presenta esa prueba y se exponen los resultados de la sección en que se evalúa metacomprensión, centrada, en este caso, en la formulación de preguntas, uno de los componentes fundamentales en el aprendizaje de estrategias comprensivas. El análisis revela que los futuros docentes tienen competencias metacognitivas bajas, ilusión de conocimiento y serias dificultades para, a partir de un texto escrito, hacer preguntas que superen la aclaración de problemas léxicos. Del diagnóstico se infieren posibles características de la enseñanza de la lectura que desarrollarán los futuros docentes.

\section{Palabras claves:}

Crisis educativa, enseñanza de la lectura, competencia metacognitiva, formulación de preguntas.

\section{Abstract}

Having on mind the existence of crises and recurrent projects for the education laws renewal, the making of diagnoses about students' and teachers' problems, among other issues, is imperative. In order to make a contribution in this sense, a team of researchers led by professor Elvira Arnoux at Lingüistics Institute, in Buenos Aires University, has designed and administrated a reading survey and a test on comprehension and metacomprehension in a postsecondary education level, which has been the research problem of a few studies: students at teachers training institutes for initial, primary and secondary schools in the province of Buenos Aires (Argentina). In the present article that test is introduced; the findings mainly exposed are the ones of the section on metacomprehension, focused in this case on question making -one of the fundamental components of the learning of reading strategies. The analysis reveals that the ones who are to become teachers have low metacognitive competence, knowledge illusion and serious difficulties to state questions on a written text, questions which go beyond the resolution of lexical problems. Some possible reading instruction characteristics, these future teachers will develop, are inferred from this diagnose.

\section{Keywords:}

Education crisis, reading instruction, metacognitive competence, question making

Artículo recibido el 20 de octubre de 2006 y aprobado el 30 de abril de 2007

* Investigadoras del Instituto de Lingüística, Universidad de Buenos Aires, Argentina. elvira@filo.uba.ar, snogueira@tournet.com.ar , adrianasilvestri@ciudad.com.ar. 


\section{Introducción}

Los institutos de formación docente son primordiales en la marcha del sistema educativo argentino en los niveles inicial, primario y secundario. De allí la importancia de elaborar diagnósticos sobre las competencias discursivas de los futuros docentes, que puedan orientar pedagógicamente a los responsables de dichos establecimientos y al cuerpo de profesores. Con ese objetivo diseñamos y administramos encuestas sobre prácticas lectoras, (Blanco et al., 2005) al igual que pruebas de comprensión y metacomprensión, en institutos de formación de docentes para los distintos niveles.

El primer procesamiento de algunos resultados obtenidos en las pruebas respondidas por estudiantes inscritos en la carrera del magisterio, centradas en la comprensión macroestructural y en las competencias de escritura necesarias para elaborar una síntesis, reveló escasas estrategias de revisión, falta de sensibilidad hacia las marcas gramaticales y discursivas -en especial las que corresponden a estrategias retóricas de la explicación- y una lectura lineal no integrativa. Incluso cuando respondían correctamente, evidenciaban limitadas habilidades de reformulación, construcción de textos poco articulados, baja cohesión e insuficiente autonomía explicativa (Arnoux, Nogueira y Silvestri, 2006). Así mismo, en las respuestas a preguntas abiertas de la encuesta de prácticas lectoras los estudiantes mostraron dificultades para desplegar un texto, desconocimiento de los requerimientos de un escrito -que se exponía en la tendencia a distribuir los segmentos en el espacio siguiendo las pautas de la oralidad-, problemas para armar oraciones, articular los segmentos internamente, citar, reconocer la concordancia, emplear la puntuación y usar en forma adecuada las mayúsculas, y dificultades ortográficas -la mitad de las pruebas tenía como mínimo un error de ortografía-, léxicas y de registro (Arnoux, 2004).

En el presente artículo, la población abordada son los alumnos que concurren al último año de las carreras de formación de profesores de secundaria. El tramo analizado de la prueba busca evaluar, a partir de la consigna de formular posibles preguntas a un docente, las habilidades metacomprensivas (Peronard, Crespo y Velásquez, 2000; López y Arciniegas, 2004). Consideramos como tales aquellas que implican un control y regulación del proceso de lectura según su finalidad y los rasgos del texto, una capacidad para reconocer las dificultades de comprensión

-frente a un texto en particular o como lector de determinado tipo de materiales, o como lector en general-, y una disposición para definir los lugares textuales que plantean problemas y las líneas de un posible avance en el conocimiento de la temática abordada. En el fragmento de la prueba que vamos a abordar, la formulación de preguntas tendía, básicamente a que los estudiantes se concientizaran del proceso de comprensión y verbalizaran con el formato de pregunta sus dificultades, dudas o necesidades de profundización del tema tratado.

\section{Formulación de preguntas}

La formulación de preguntas es un proceso fundamental para la ciencia cognitiva y la educación, ya que se trata de uno de los componentes claves de la comprensión, la resolución de problemas, el razonamiento y la creatividad (Otero y Graesser, 2001).

En el terreno educativo, se considera crucial para el aprendizaje de estrategias comprensivas, de modo que numerosos métodos de enseñanza se basan en el entrenamiento para que el estudiante aprenda a formular preguntas productivas (Palincsar y Brown, 1984). Por una parte, la pregunta interviene en los momentos iniciales del aprendizaje, cuando la actividad de comprender un texto se realiza en forma compartida entre el docente y el alumno; en este caso, las preguntas se encaminan a obtener información para resolver problemas de superficie (por ejemplo, los generados por el desconocimiento léxico) o de la base textual, es decir, la información explícita del texto y las inferencias simples que permiten resolver la coherencia.

Pero, además, la pregunta es un recurso eficaz para lectores expertos, a quienes la superficie y la base textuales no les presentan dificultades, pero que se proponen profundizar la comprensión, relacionar e integrar sus conocimientos con la in- 
formación provista por el texto. En este sentido, la pregunta proporciona información sobre el nivel de representación del escrito alcanzado por el lector (Keyes, 1996; Otero y Graesser, 2001). Incluso los buenos lectores, con elevado conocimiento temático, continúan empleando el recurso de la pregunta, ya sea formulada a otro experto o autoformulada, puesto que una de las competencias del experto es la habilidad para buscar información pertinente, ya sea en el mismo texto leído o en otros.

A pesar de la indiscutida valoración de la pregunta y de las abundantes investigaciones que demuestran sus beneficios para el aprendizaje, se ha observado que el estudiante estándar -niño, adolescente o adulto- formula escasas preguntas al docente, en promedio una por hora de clase, y se trata además de preguntas inmaduras, que poco ayudan a resolver problemas comprensivos (Graesser et al., 2005). Dos factores que podrían explicar esta situación son, por una parte, la falta de entrenamiento específico para la formulación y, por otra, la baja calidad de las preguntas que los docentes hacen en clase, proporcionando así a sus alumnos un modelo pobre (Graesser et al., 1996).

En general, los estudiantes sostienen un supuesto implícito según el cual la pregunta es un proceso automático, que "surge" espontáneamente (Greene, 2005). Consideran, además, que la pregunta es apenas un procedimiento de clausura de dudas y no de profundización de conocimiento. Estas creencias erróneas son compartidas a menudo por el docente o generadas de manera implícita por su actividad en el aula.

La habilidad para hacer preguntas comprende componentes cognitivos y lingüísticos complejos, que deberían contemplarse en un buen entrenamiento específico. Por una parte, el lector debe disponer de habilidades verbales satisfactorias para formular una pregunta de modo comprensible y preciso. Por otra, entre las operaciones cognitivas requeridas para dicha formulación se encuentran la búsqueda y comparación de información, la detección de discrepancias en el texto y entre el texto y el conocimiento previo, el razonamiento analógico, el conocimiento pragmático y, en especial, las habilidades metacognitivas del lector (Graesser et al., 2005).

Para formular una "buena" pregunta se requiere, en efecto, disponer de una serie de habilidades que suponen actividad metacognitiva: la evaluación adecuada de la propia comprensión, la identificación de la fuente del problema y la valoración de la pregunta como recurso que permite resolver problemas y profundizar conocimientos.

Como es sabido, las habilidades metacognitivas son de desarrollo tardío (Gombert, 1990; Mateos, 2001), de modo que es de esperar que los lectores más jóvenes hagan preguntas inmaduras, que revelan escasa o nula competencia en este aspecto. En cambio, cuando esta inhabilidad se detecta en sujetos mayores, se puede atribuir al escaso conocimiento previo sobre el tema o a una enseñanza insatisfactoria (Graesser et al., 2005).

Así, las preguntas que los lectores inexpertos formulan tienden a ser confusas, de pobre control lingüístico, más breves y poco contextualizadas, muy genéricas, aplicables a todo texto debido a que no apuntan directamente a un contenido específico, o contestadas en el texto, lo que revela una lectura desatenta o una falta de relectura. Además, es infrecuente que se refieran a aspectos formales o retóricos del mismo, que se citen fragmentos del texto, o que se busquen definiciones concretas de conceptos abstractos (Greene, 2005).

Diversas investigaciones ratifican estas características en las preguntas de los alumnos adolescentes y adultos (Graesser et al., 2005; Silvestri, en prensa). Como señalamos, la modelización por parte del docente resulta crucial para desarrollar esta habilidad, de modo que se consideró pertinente indagarla en estudiantes de carreras docentes 1 .

Para organizar en el aula actividades tendientes al aprendizaje de estrategias de comprensión, es necesario que el docente conozca los componentes

1 Esta investigación se inscribe en un proyecto más amplio, "Hacia una enseñanza sistemática de la lectura y la escritura en el nivel medio y en el primer año de los institutos de formación docente en zonas con población vulnerable de la Ciudad Autónoma de Buenos Aires y del Conurbano Bonaerense", UBACyT 2004-2007, dirigido por Elvira Narvaja de Arnoux. 
del proceso y sepa cómo regularlo con eficiencia. Esta competencia metacomprensiva debería observarse, en primer lugar, en la gestión que el docente realiza de sus propios procesos comprensivos, en las estrategias que pone en juego ante una dificultad o ante el deseo de profundizar el conocimiento. Un docente que, en su papel de estudiante lector, no revela habilidad para formular preguntas, en modo alguno será capaz de ofrecer a sus alumnos un modelo satisfactorio o de planear actividades para desarrollar esta habilidad.

El objetivo de este trabajo es, entonces, analizar las habilidades metacomprensivas de los estudiantes de profesorado, en particular su competencia en la formulación de preguntas que ayudan a solucionar problemas de comprensión o a profundizarla. Se eligieron grupos de estudiantes próximos a egresar $y$, por lo tanto, a desempeñarse como docentes, de modo que este análisis puede revelar qué estrategias de comprensión están en condiciones de implementar en su trabajo en el aula.

\section{Metodología}

Si bien el trabajo en el aula -en lo que a la formulación de preguntas respecta- podría favorecer la elección del discurso oral de los futuros docentes, se optó por el discurso escrito. Esta elección se basó, por una parte, en las ventajas metodológicas que proporciona el procesamiento de material escrito, mientras que por otro lado, Graesser et al. (1996) señalan que los estudiantes formulan oralmente sólo el $10 \%$ del número de preguntas que pueden llegar a hacer por escrito, ya que es difícil superar el costo social de exponer el desconocimiento, de modo que se consideró más productiva la modalidad escrita. Finalmente, esta modalidad brinda posibilidades de reflexión y relectura, importantes componentes de las habilidades metacognitivas que se están evaluando.

\section{Sujetos}

Participaron en el trabajo 65 estudiantes de $4^{\circ}$ año del profesorado para EGB 3 y Polimodal de la provincia de Buenos Aires. La muestra se consideró representativa, puesto que se incluyeron profesora- dos de asignaturas variadas en establecimientos de nueve distritos?

\section{Materiales y procedimientos}

Los alumnos resolvieron en forma anónima una prueba diseñada con diversos objetivos, algunos de los cuales no se desarrollarán en este trabajo.

En la prueba (ver apéndice) hay en primer lugar un texto titulado “¿Qué es el constructivismo?", representativo de los textos habituales en las carreras de formación docente. El original se adaptó mínimamente: las modificaciones se limitaron a acentuar la organización textual original, que cuenta con marcadores discursivos ("primero", "segundo", "tercero") que ordenan "las características esenciales a toda posición constructivista". Los párrafos se numeraron para facilitar las respuestas a las consignas. La dificultad más evidente que podían enfrentar los estudiantes era el alto grado de abstracción de los conceptos, uno de los principales factores que obstaculizan la comprensión (Freedle, 1997). Sin embargo, el texto en cuestión no presentaba dificultades mayores que las de cualquier texto en el que se definen conceptos básicos de estudios introductorios del nivel superior.

En la prueba se solicitaban primero datos de la institución, la localidad en la que está asentada la escuela, el año y turno que cursa el alumno, el año en que finalizó los estudios secundarios y la edad. A continuación, se presentaba un cuestionario de comprensión sobre el texto, formulado para evaluar diferentes hablidades, tales como comprensión macroestructural, reformulación y conocimiento previo.

Para los fines de este trabajo, el desempeño de los participantes en la prueba de comprensión se tomó en forma global, asignándole un puntaje máximo de 10 (diez) puntos, con el objeto de relacionar el puntaje obtenido con el desempeño en la segunda parte de la prueba.

En esta segunda parte, los sujetos debían resolver una serie de consignas destinadas a analizar sus

2 Los distritos representados fueron Moreno, Azul, Olavarría, Ituzaingó, San Nicolás, Ramallo, Villa Constitución, Baradero y Dolores. 
habilidades metacomprensivas. En la primera se solicitaba evaluar en una escala de cinco grados la dificultad del texto leído; en la segunda se proponía indicar cuál párrafo había resultado más fácil y cuál más difícil de comprender, y explicar por qué. Finalmente, la tercera consigna era “ $¿ Q$ Qué preguntas le haría al docente para comprender mejor el texto?". Se consideró que la resolución de la segunda consigna ayudaría a los participantes a prestar atención a las dificultades concretas que la lectura del texto pudiera plantearles y, por lo tanto, podría funcionar como facilitador para la formulación de las preguntas subsiguientes.

El tiempo asignado a la tarea fue de 40 minutos. Todos los instrumentos los administraron en sus respectivos institutos los docentes que participaban en el proyecto ${ }^{33}$, quienes en muchos casos contaron con la colaboración de otros colegas.

\section{Resultados y discusión}

\section{Evaluación de la dificultad del texto}

En la prueba de comprensión, los estudiantes obtuvieron una media de 5,04/10 puntos. A pesar de este desempeño poco satisfactorio, el 74\% evaluó la comprensión del texto entre "muy fácil" y "normal" y sólo el 7,5\% la consideró "difícil" o "muy difícil”, como puede observarse en el gráfico siguiente.

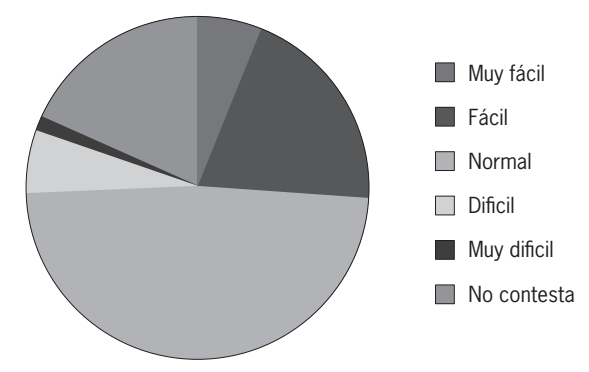

Gráfico 1. Evaluación de la dificultad del texto (porcentaje de alumnos)

3 En el marco del proyecto se organizó, a partir de una convocatoria de las autoridades educativas de la provincia de Buenos Aires, un seminario con 25 profesores de institutos de formación docente, destinado a elaborar un diagnóstico sobre la situación de los institutos y a realizar propuestas de cambio curricular y actualización de los docentes de la enseñanza superior
Se advierte, además, que un número no desdeñable (18\%) no resolvió la consigna.

Esta imposibilidad de evaluar la dificultad de lectura se consideró indicadora de habilidades metacomprensivas bajas. Se incluyó también en este grupo a los estudiantes que evaluaron el texto como "fácil" o "normal", a pesar de haber obtenido en la prueba de comprensión un puntaje muy bajo, igual o menor a $3 / 10$ (18\%), o a quienes combinaron el puntaje bajo con una o más preguntas sin resolver (25\%). Estos dos últimos casos evidencian la situación descrita como "ilusión de conocimiento", en la que el sujeto cree haber comprendido aun cuando esta creencia es errónea. La "ilusión de conocimiento" se presenta en el nivel más bajo de competencias metacognitivas, pues la imposibilidad de evaluar el propio proceso comprensivo impide la puesta en marcha de mecanismos de control o de reparación (Ehrlich, 1997). En total, entonces, el 54\% de los estudiantes evidenció habilidades metacomprensivas bajas.

\section{Evaluación de la dificultad de los párrafos}

De los 65 alumnos evaluados, 19 (29\%) no respondieron ningún punto de la segunda consigna que evaluaba la metacomprensión, la que solicitaba que señalaran qué párrafo les había resultado más fácil, cuál más difícil y que explicaran por qué en cada caso. Otros cuatro alumnos llegaron a identificar los párrafos en cuestión, pero no presentaron explicaciones de su selección, lo que eleva a un 35\% el promedio de alumnos que no dieron cabal cuenta de su propio proceso de lectura. Para la identificación, un conocimiento implícito y una percepción difusa de ese proceso serían suficientes; por el contrario, la explicación demanda un mayor grado de conciencia, que permita el pasaje de lo implícito a lo explícito, de la imprecisión a la precisión de la formulación verbal. A estos números se suman otros dos casos que eligen todos los párrafos para los dos puntos de la consigna, con explicaciones del tipo "Ningún párrafo me resultó más sencillo/difícil que otro”.

Además, entre los estudiantes que señalaron párrafos del texto tal como se les demandaba, 45 indicaron el más fácil para su comprensión y 32, el 
más difícil; sólo uno señaló el párrafo más difícil, sin indicar el que le había resultado más fácil. Estos datos corroboran que la operación de toma de conciencia de la dificultad resulta más compleja en el proceso de desarrollo metacognitivo.

De esta manera, el 58\% de los alumnos no resolvió por completo la consigna en cuestión, cifra próxima al 54\% que evidenció habilidades metacomprensivas bajas a propósito de la evaluación global del texto.

Como se observa en los gráficos presentados a continuación, el párrafo seleccionado por el número de alumnos como el más fácil de comprender fue el segundo y como el más difícil, el tercero.

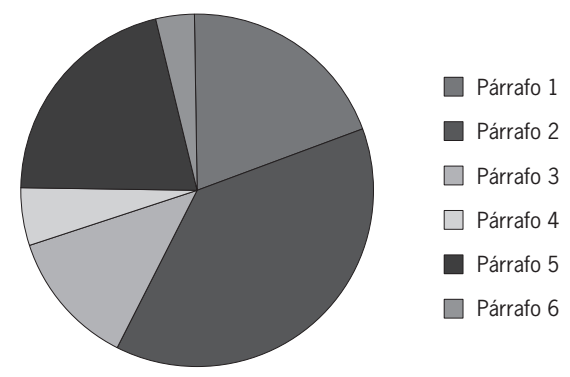

Gráfico 2. Párrafo señalado como el más fácil (porcentaje de alumnos)

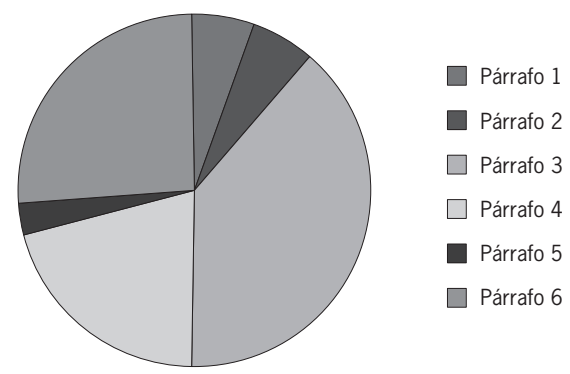

Gráfico 3. Párrafo señalado como el más difícil (porcentaje de alumnos)

Cabe anotar que los dos párrafos señalados por mayor cantidad de estudiantes como el más fácil y el más difícil corresponden a una misma sección del texto: la explicación de la primera característica el constructivismo. El segundo párrafo, el más fácil para la mayoría, describe el constructivismo contrastivamente, en "oposición a concepciones conductistas e innatistas". El tercero expande esa oposición e introduce otro contraste, esta vez entre posiciones en el interior del constructivismo. Podría deducirse que si bien la comparación contrastiva les resulta a estos alumnos un facilitador de la comprensión, el hecho de que el texto sostenga esa misma operación -pero a propósito de diversos subtópicos y dentro de un mismo párrafo- les dificulta la lectura.

- Alumno 579:

- (Sobre el 2 como el párrafo más fácil): Se produce una aproximación al constructivismo de una manera clara y con lenguaje sencillo.

- (Sobre el 3 como el más difícil): Se pone en juego más de una idea en el mismo párrafo.

La dificultad que para los lectores poco entrenados plantean las equivalencias parciales en la construcción de un texto, en este caso, la continuidad de una operación discursiva con variación de subtópico, otros estudiantes la señalan en modos como el siguiente:

- Alumno 580 (el párrafo 3): Tiene varios conceptos importantes, que a simple lectura parecen explicar lo mismo y no lo es.

La cuestión léxica es importante como razón del señalamiento del tercer párrafo como el más difícil: "epistemológicas" sobresalió como término desconocido para los alumnos y se indicó como motivo de dificultad de comprensión también del último párrafo, en el que hay otra ocurrencia de ese adjetivo. El sexto párrafo, como puede verse en el gráfico 3, fue el más difícil para estos estudiantes después del tercero. Aparecen justificaciones como éstas:

- Alumno 577: Me resultó difícil porque no tengo claro el concepto de "epistemología".

- Alumno 598: No define "asuntos epistemológicos"...

El sexto párrafo genera también dificultades a estos estudiantes porque demanda resolver una correferencia textual. Un solo alumno comprende con precisión el problema, los demás no llegan a hacer la relectura necesaria siquiera para citar el fragmento correctamente: 
- Alumno 539: Hace referencia a "los aspectos reseñados antes" y estoy cansada para releer y entender cuáles son esos aspectos.

- Alumno 561: Porque no se evidencia fácilmente cuáles son "las dos etapas" de las que habla.

Los conocimientos previos, que los alumnos señalan como factor que les habría facilitado la comprensión del segundo párrafo, no les resultan suficientes para resolver los problemas con el léxico o reconocer la coherencia entre ese párrafo y el tercero, por ejemplo a propósito del conductismo:

- Alumno 584 (sobre el 2, como el más fácil): Porque tengo conocimiento de las distintas concepciones (conductistas e innatistas), ya que las he estudiado en el profesorado.

(Sobre el 3): Por la terminología que utiliza.

- Alumno 538 (sobre el 2): Es el que me resultó más familiar, porque esa es la idea principal del constructivismo.

(Sobre el 3): Es el más confuso, el conductismo como posición epistemológica. No me acordaba que fuera así.

Al hablar de sus conocimientos previos, algunos estudiantes disocian explícitamente sus aprendizajes conceptuales de los léxicos. Un alumno que justifica su selección del párrafo más fácil dice:

- Alumno 553: Porque al haber cursado psicología años anteriores, el vocabulario y el concepto me es conocido, por lo cual es fácil de comprender.

Entre los estudiantes que no resolvieron la consigna de la evaluación de los párrafos del texto o lo hicieron parcialmente, el 58\% obtuvo una nota inferior a 6 -calificación que promociona en el sistema educativo no universitario en Argentina- en la parte de comprensión de la prueba; entre los que completaron la consigna en cuestión -más allá de si lo hicieron correctamente-, ese porcentaje bajó a 44 . Se observa en este punto, entonces, una correlación positiva entre la comprensión de los estudiantes y su disposición a reflexionar sobre el texto y sobre el propio proceso de comprensión, aunque no puedan realizar esta actividad fluidamente o con precisión, como se evidenció en las explicaciones que dieron.

\section{Clases de explicaciones de los alumnos sobre la evaluación de los párrafos}

Entre las explicaciones que presentaron los estudiantes de su selección de los párrafos más fácil y más difícil del texto, distinguimos las inadecuadas de las adecuadas. Entre las primeras, incluimos:

- Las que no se ciñen a lo solicitado por la consigna

- Observación: ningún párrafo me resultó más sencillo que otro. Observación: ningún párrafo me resultó más difícil que otro.

- Las tautológicas o que se aproximan a formas tautológicas en función de la consigna (indicar cuál párrafo había resultado más fácil y cuál más difícil de comprender, y explicar por qué)

- (Sobre el 2 como el más fácil): Porque se podía comprender lo que el autor quería explicar.

- (Sobre el 4 como el más difícil): No entiendo bien los conceptos.

- Las que son imprecisas

- (Sobre el 1 como el más difícil): Me resultó más difícil porque la idea está implícita.

- Las que presentan algún error conceptual

- (Sobre el 6 como el más difícil): Porque no se evidencia fácilmente cuáles son "las dos etapas" de la que habla.

Los resultados evidenciaron, también en este punto, que la toma de conciencia sobre la dificultad es más compleja. Diez (22\%) de las cuarenta y cinco explicaciones del señalamiento del párrafo más fácil fueron respuestas inadecuadas; en este caso predominaron las explicaciones imprecisas (seis de esas diez). Doce (37\%) de las treinta y dos explicaciones sobre el párrafo más difícil resultaron inadecuadas; dos tipos de justificaciones dominaron en este grupo: las imprecisas y las que presentaron algún error conceptual. Como se observa en el ejemplo recién citado de explicación con algún error de ese tipo, las habilidades metacomprensivas de estos estudiantes les permiten intuir segmentos del texto con los que 
tienen dificultad, pero no identificar sus problemas, a partir de lo cual podrían reajustar su proceso de comprensión. En el caso citado, el alumno no detecta que está reemplazando el "aspecto (del constructivismo)" que aparece en el sexto párrafo del texto por "etapas", reemplazo que puede hacerlo buscar sucesiones temporales que la fuente no plantea.

En las respuestas imprecisas se asoció "fácil" con "claro" y "sencillo" y "difícil" con "confuso", "no claro" y "no fácil", sin avanzar más en la explicación solicitada. En las que presentaron errores conceptuales, se pone en evidencia que los estudiantes no detectan definiciones y paráfrasis incluidas en el texto, o atribuyen a una misma postura epistemológica rasgos que la fuente asigna a distintas posiciones.

Las respuestas adecuadas retomaron mayoritariamente dichas asociaciones, pero de algún modo especificaron en qué consistía la "claridad", la "sencillez" o sus contrapartes:

- (Sobre el párrafo 2 como el más fácil): Porque es el que más deja en claro las características que yo manejo del constructivismo.

- (Sobre el 4 como el más difícil): No es claro. Los conceptos merecerían más desarrollo.

La facilitación de la comprensión se asocia mayoritariamente en estas respuestas con la apelación a conocimientos temáticos previos que el lector posee, el léxico "no específico" y la exposición de diferencias. En menor grado se señalaron operaciones discursivas como la ejemplificación y el discurso metatextual (la partitio del primer párrafo). La obstaculización del proceso de comprensión, correlativamente, se vinculó a la falta de conocimientos previos por parte del lector -temáticos y léxicos-.

Las explicaciones adecuadas pueden subclasificarse, atendiendo a qué cuestiones ligadas a la comprensión abordaron:

- Operaciones o estrategias propias del proceso de comprensión.

- Factores textuales que facilitan o dificultan la comprensión.

Las explicaciones que apelaron a estrategias propias del proceso de comprensión fueron domi- nantes, en especial las que apuntaron a la incidencia del conocimiento previo sobre el tema. Entre las respuestas sobre el párrafo más fácil, un 36\% explicó que ya había estudiado el constructivismo; entre las referidas al segmento más difícil, un $32 \%$ señaló que le faltan saberes para comprenderlo o que lo expuesto por el texto contradice lo que ya se sabía:

- (Sobre el 2 como el más fácil): Necesitaba establecer o reconocer las diferencias entre estas corrientes, ya que se mezclan a diario en mis conocimientos.

- (Sobre lo mismo): Este tema ya lo había visto en materias pedagógicas y además porque generalmente las teorías surgen por oposición unas a otras y porque la postura constructivista requerirá, sin duda, un sujeto "constructor activo".

- (Sobre el 4 como el más difícil): Por no tener en claro algunos conceptos aprendidos pero ya olvidados.

- (Sobre el 5): Me resultó más difícil debido a que los dos primeros filósofos los conocía, pero al tercero, Maturana, nunca lo había escuchado.

Los alumnos identificaron también la necesidad de relectura y la incidencia de factores mentales que intervienen en el proceso de comprensión:

- (Sobre el 6 como el más difícil): Hace referencia a "dos aspectos reseñados antes" y estoy cansada para releer y entender cuáles son esos dos aspectos.

- (Sobre el 1 como el más fácil): Porque me llamó la atención de no encontrar las 3 ctes. que el autor nos iba a describir. Porque le tuve que prestar más atención.

Entre los factores textuales que facilitan o dificultan la comprensión, se señaló frecuentemente el léxico:

- (Sobre el 1 como el más fácil): Porque muestra una visión global de los puntos a tratar luego y hay ningún término específico.

- (Sobre el 3 como el más difícil): Por el vocabulario.

Así mismo, se señalaron cuestiones de sintaxis y operaciones discursivas: 
- (Sobre el párrafo 4 como el más fácil): Las oraciones resultan sencillas, con significados claros y precisos.

- (Sobre el 1 como el más fácil): Porque acota el área de estudio y anuncia el tema.

Finalmente, a propósito de las habilidades metacomprensivas bajas puestas en evidencia en la consigna sobre la evaluación de los párrafos del texto, cabe señalar que de los 27 alumnos que resolvieron completa esa tarea, apenas uno señaló dos causas que justificaran su elección de los párrafos más fácil y más difícil para su comprensión. El resto, en general, sólo expuso un motivo.

\section{Preguntas formuladas}

La consigna “¿Qué preguntas le haría al docente para comprender mejor el texto?” obtuvo una escasa cantidad de propuestas ${ }^{44}$, en promedio menos de una pregunta por alumno $(0,9)$, distribuidas tal como se observa en el gráfico 2.

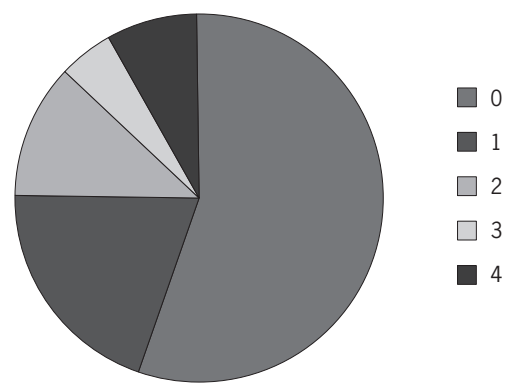

2. Gráfico 2. Cantidad de preguntas formuladas (porcentaje de alumnos)

Vemos que el grupo mayor (55\%) no formuló ninguna pregunta, seguido por un $20 \%$ con una pregunta y un $12 \%$ con dos preguntas. Así las cosas, apenas el 13\% hizo entre tres y cuatro preguntas.

Sólo dos estudiantes creyeron necesario aclarar por qué no formulaban ninguna pregunta. Uno de los casos fue el de uno de los participantes incluido en el subgrupo de habilidades metacomprensivas

4 Si bien la consigna solicitaba preguntas, muchos estudiantes optaron por enunciados de función similar, tales como la solicitud de aclaración o explicación. Se consideraron válidas todas las opciones reformulables en forma de pregunta. bajas, ya que su desempeño en la prueba de comprensión fue de 3 (tres) puntos. Pese a este resultado, el estudiante consideró que había comprendido:

- Creo que ninguna [pregunta], ya que es la 6ta. vez que leo el texto completo. Pude darme cuenta de palabras o conceptos que al comienzo no lograba descifrar.

En el segundo caso, el alumno tuvo un desempeño promedio de 6 (seis) puntos. Su justificación fue la siguiente:

- Realmente creo que el texto se comprende bien, no se necesita más ayuda que la brindada por la guía.

En ambos casos puede advertirse una concepción restringida del proceso de comprensión, que se limita a la base o incluso la superficie del texto (comprender "palabras" o "conceptos", como se señala en el primer ejemplo) en lugar de advertir que la pregunta es además un recurso valioso -como ya señalamos- para profundizar el conocimiento.

En cuanto al desempeño en la prueba de comprensión, no se observó ninguna correlación entre el puntaje obtenido y el número de preguntas, resultado que coincide con el de otras investigaciones (Graesser et al., 2005). No obstante, los alumnos con habilidades metacomprensivas bajas formularon en promedio la mitad de preguntas que el subgrupo de habilidades estándar (0,6 preguntas por alumno vs. 1,2).

Otero y Graesser (2001) proponen dos hipótesis complementarias que podrían explicar la escasez de preguntas. Por una parte, el número de preguntas se encuentra en relación con el conocimiento previo, de modo que cuanto más escaso es éste, menos preguntas se pueden formular. Así, paradójicamente, es necesario disponer de una cantidad significativa de conocimientos sobre el tema en cuestión para "saber lo que uno no sabe" y para poder enunciar este desconocimiento de manera que conduzca a subsanarlo. En el caso del grupo estudiado, ésta puede ser una de las razones que explican la baja cantidad de preguntas. 
Por otra parte, el conocimiento previo poco profundo puede conducir a la formulación de mayor númeno de preguntas, pero inmaduras, hipótesis que trataremos en el próximo punto.

\section{Clases de preguntas formuladas}

En el gráfico 3 puede observarse la frecuencia de las distintas clases de preguntas formuladas

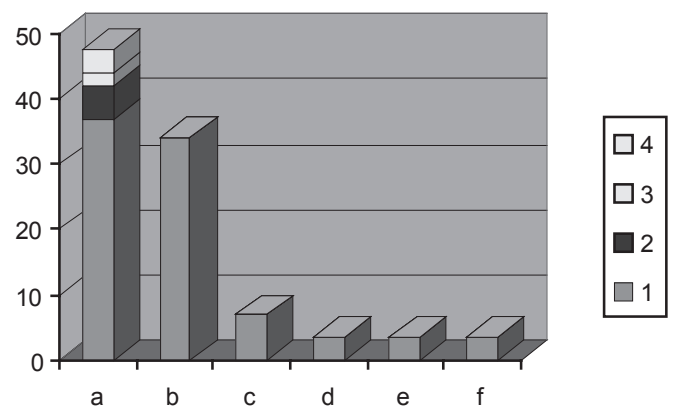

Gráfico 3. Clases de preguntas (porcentaje sobre el total de preguntas formuladas).
a: formulación literal
b: solicitud de ampliación
c: solicitud inespecífica de aclaración
d: no contestable
e: aspectos formales
f: solicitud de operaciones

a.1. en relación con el cuestionario, con respuesta en el texto

a.2. en relación con el cuestionario, sin respuesta en el texto

a.3. sin relación con el cuestionario, con respuesta en el texto

a.4. sin relación con el cuestionario, sin respuesta en el texto

A continuación, se analizará y ejemplificará cada tipo de respuesta, en orden de frecuencia.

a) Preguntas sobre una expresión literal de la fuente El 48\% de las preguntas indagó sobre aspectos superficiales del texto, es decir, sobre una palabra o expresión tal como aparece en el mismo o apenas pa- rafraseada. Entre éstas, el 12,5\% fue de orden léxico (la mitad sobre la palabra "epistemológico").

En el siguiente ejemplo se advierte esta clase de operación:

Fuente: (...) toda posición constructivista rescata el rol constructivo del sujeto (...)

Pregunta: Explique el rol constructivo del sujeto.

Debe señalarse que, entre estas preguntas, la mayor parte (a.1.y a.3., el 81\% dentro de este tipo) solicitaba respuestas que se encontraban en el texto o eran fácilmente inferibles a partir de la información proporcionada por él. En muchos de los casos (a.1.), la pregunta solicitaba respuesta para alguna consigna del cuestionario de comprensión, operación muy inmadura que busca que el docente resuelva la respuesta, en lugar de solicitar información para contestarla en forma autónoma:

Cuestionario: ¿Cuáles son las tres corrientes del constructivismo a las que se refiere el autor?

Pregunta: ¿Cuáles son las corrientes que el cuestionario pregunta?

Mucho menos frecuente fue el caso en que la pregunta no dependía del cuestionario (a.3. y a.4., $11 \%$ dentro de este tipo), evidenciando una construcción comprensiva más independiente, capaz de orientarse hacia segmentos del texto que no se subrayan en el cuestionario. Aun así, recordemos que para la mitad de estas preguntas (a.3.) la respuesta se encontraba en el texto. El siguiente es un ejemplo del subgrupo de preguntas a.4., las más maduras dentro de esta clase:

Pregunta: ¿Por qué los conductistas e innatistas consideran al sujeto como inexistente?

\section{b) Preguntas que solicitan ampliación}

El 34\% de las preguntas pertenece a este grupo que, en principio, es el que debería haber predominado en estudiantes de este nivel, puesto que se trata de preguntas que presuponen que la base del texto se ha comprendido en forma satisfactoria y se intenta profundizar el conocimiento. Sin embargo, en muchos casos, aunque el sujeto pide explícitamente mayor información, ésta es relativa 
a elementos de la base del texto, de modo que esta clase de pregunta puede considerarse un híbrido entre el grupo anterior y las solicitudes legítimas de ampliación. Los dos ejemplos siguientes muestran ambas posibilidades:

Pregunta por la base más ampliación: ¿Qué es la epistemología? Ampliar.

Ampliación legítima: ¿Cómo se aplica el constructivismo en el aula?

c) Preguntas inespecíficas que solicitan aclaración Esta clase de pregunta revela una construcción conceptual difusa y también escasa capacidad para la formulación precisa, características del lector inmaduro. Una pregunta de esta índole (cuya forma menos desarrollada es el "no entendî") puede implicar que el lector no comprendió casi nada o que es incapaz de formular verbalmente una duda concreta, como se observa en estos ejemplos:

- Le pediría que me aclarara el párrafo 6 .

- Explicar las diferentes posturas de los distintos autores.

En estos casos, el docente no puede proporcionar respuestas focalizadas, sino -ante la falta de información sobre qué es lo que realmente no se comprendió- intentar una explicación de todo o casi todo el texto.

d) Preguntas incomprensibles o formuladas con error conceptual

Un pequeño porcentaje $(3,5 \%)$ está integrado por preguntas que no se pueden contestar, en tanto incluyen un error de concepto o presentan una formulación muy confusa:

- El easo objeto de estudio es el constructivismo o el sujeto cognitivo influenciado por un constructivismo.

- ¿Qué tipo de acepciones o posiciones rescata el constructivismo?

En el primer caso, en el que el tachado revela un intento por encontrar el término más adecuado, no se logra siquiera una formulación de pregunta o de pedido de explicación. Al mismo tiempo, se incluye una presuposición errónea (que el constructivismo "influencia" al sujeto), lo que hace que el presunto interrogante se vuelva imposible de responder. En el segundo ejemplo, las suposiciones falsas son que el constructivismo rescata "acepciones" o que "acepciones" es sinónimo de "posiciones".

e) Preguntas por aspectos formales del texto

También escasa en este corpus (3,5\%), esta clase de pregunta puede llegar a convertirse en característica de un lector maduro, siempre y cuando sea pertinente. El lector inmaduro, como señalamos, no suele atender a aspectos retóricos o formales del texto. Sin embargo, es posible que advierta algún aspecto irrelevante que no contribuye a la comprensión. Los siguientes casos ejemplifican ambas posibilidades:

Pertinente: ¿No convendría ubicar el párrafo cinco (adaptándolo) en el uno para que no queden tan lejos las tres corrientes?

No pertinente: ¿Por qué enumerar los párrafos?

En el ejemplo pertinente -más allá de acordar con la modificación sugerida- el alumno propone, para favorecer la comprensión, un cambio en la organización de contenido del texto que revela una correcta asociación entre los conceptos desplegados en el primero y el quinto párrafos. En cambio, la numeración de párrafos que llama la atención del estudiante en el segundo ejemplo no está vinculada a operaciones de comprensión, sino que se introdujo para facilitar las respuestas al cuestionario, lo que debería haber sido obvio al leer las consignas.

e) Solicitud de operaciones

Otro porcentaje mínimo del 3,5 correspondió a preguntas que solicitaban del docente operaciones como la ejemplificación o el resumen sinóptico, tal como se observa en los ejemplos:

- Desarrolle un cuadro comparativo entre las tres corrientes del constructivismo.

- ¿Por qué el constructivismo se opone al conductismo? Ejemplifique. 
En el primer ejemplo, la solicitud es pertinente, pero no así en el segundo, ya que la causa de una diferencia teórica no resulta ejemplificable. De todos modos, llama la atención la escasez de preguntas de este tipo entre futuros docentes, quienes deberían tener muy presente esta clase de recursos como facilitadores de la comprensión. Como ya señalamos, el texto empleado se caracteriza por su alto grado de abstracción, y la forma adecuada de facilitar la comprensión de un concepto abstracto es, obviamente, una operación de concreción, como proporcionar ejemplos. Pero sólo una pregunta los solicitó, y ésta de manera poco pertinente.

\section{Conclusiones}

Numerosos diagnósticos han señalado, en los últimos años, el nivel poco satisfactorio que se advierte en las competencias de los alumnos de enseñanza media para la comprensión lectora y, por ende, para el aprendizaje a partir de textos (Martínez et al., 2004; Parodi, 2005).

A pesar de que la intuición de los investigadores y la experiencia de los docentes de nivel terciario sugería que uno de los factores centrales que provocan esta situación es la formación ineficiente de los nuevos profesores, el trabajo en este nivel es todavía escaso.

Por una parte, los alumnos de los institutos de formación docente son esos mismos estudiantes de nivel secundario que egresan sin haber alcanzado una competencia comprensiva satisfactoria; por otra, dichas instituciones no siempre implementan técnicas específicas de enseñanza que permitan solucionar este problema.

Como se observa en los resultados de esta investigación, la mayoría de los estudiantes próximos a egresar revela escasas habilidades comprensivas y metacomprensivas. Más de la mitad se encuadró en el segmento de competencias metacognitivas bajas, revelando incluso "ilusión de conocimiento": si no pueden evaluar su propia comprensión, es difícil imaginar que puedan evaluar la de sus alumnos.

En cuanto al desempeño específico en la formulación de preguntas, del escaso número obtenido puede deducirse que a lo largo de su formación no se les indujo a tomar conciencia de la enorme importancia de este recurso para el propio aprendizaje $y$, por ende, para la enseñanza. Recordemos que el grupo mayoritario -más de la mitad- no formuló ninguna pregunta, a pesar de que el rendimiento promedio en la prueba de comprensión mostraba que habían surgido dificultades

-no detectadas en muchos casos- durante la lectura.

La calidad de las preguntas formuladas plantea también posibilidades poco deseables para el trabajo en el aula. Los futuros docentes mostraron una concepción estrecha sobre la comprensión, pues la mayor parte de sus preguntas fue relativa a la base del texto. En muy pocos casos se intentó asociar la información del texto con otros conocimientos del lector ni profundizar los contenidos. La pregunta superficial, por supuesto, es necesaria en un nivel inicial del aprendizaje de la comprensión o en el acceso inicial a un tema o dominio del conocimiento. Pero la escasez de preguntas más profundas muestra que, una vez resueltos los problemas iniciales, estos futuros docentes no ofrecerán a sus alumnos un modelo más complejo sobre el aprendizaje a partir de textos, sino que sus preguntas - como señala Greene (2005) - se limitarán a aclarar dudas sobre el significado de algunos términos, llevando así a la idea de que comprender es sólo acceder a la información explícita.

Por otra parte, hemos visto que casi el $40 \%$ de las preguntas estaban respondidas en el texto, lo que indica que los lectores no hacen uso de estrategias de relectura o las desconocen y, por consiguiente, no están en condiciones de enseñarlas.

Como ya se señaló, el bajo conocimiento previo sobre el tema del texto puede inducir a la formulación escasa o inmadura de preguntas, de modo que podría suponerse que, en el aula, con textos más sencillos, de contenidos que el docente domina, la calidad de las preguntas podría incrementarse. Sin embargo, no deja de ser alarmante suponer escaso conocimiento previo en estudiantes próximos a egresar sobre un tema como el constructivismo, elegido por encontrarse muy presente en las asignaturas pedagógicas y psicológicas. 
Además, cierto nivel de dificultad del texto, que no se encuentre más allá de la zona de desarrollo próximo del lector, no obstaculiza la comprensión, sino que promueve la optimización de las estrategias comprensivas y metacomprensivas (Kintsch, 1994). Así, puede preverse que frente a un texto más sencillo, para uso de los alumnos, el docente inexperto no alcance a percibir los aspectos potencialmente problemáticos para los estudiantes $\mathrm{y}$, por ende, sea incapaz de focalizar estos aspectos por medio de preguntas.

Teniendo en cuenta que en breve estos estudiantes se encontrarán ejerciendo la docencia, la posibilidad de que sus alumnos aprendan con ellos a comprender un texto, tanto a través de la instrucción directa y explícita como de manera informal, resulta muy improbable. Parece haberse instalado un círculo vicioso por el que los alumnos que egresan del nivel medio con escasas habilidades comprensivas e ingresan a la carrera docente, transitan por ella sin haber incrementado sensiblemente estas habilidades y, así, pasan a "formar" nuevas promociones que tampoco llegarán a desarrollarlas. Es evidente, entonces, que deben implementarse en los institutos de formación docente, en todas las carreras, intervenciones que se encaminen a promover en los estudiantes buenas competencias comprensivas y metacomprensivas, imprescindibles para ellos y para sus futuros alumnos. Џ

\section{Apéndice}

\section{¿Qué es el constructivismo?}

1. El concepto de constructivismo tiene multiplicidad de acepciones y connotaciones en ciencias sociales y filosofía. Más que entrar en grandes discusiones filosóficas, acotaremos su tratamiento a un ámbito muy preciso: el de la psicología y la educación. Aún más, dentro de la psicología, queremos restringirnos a tres corrientes que nos parecen las más influyentes en el discurso educativo. En nuestra opinión, éstas son las características esenciales de toda posición constructivista.
2. Primero: toda posición constructivista rescata al sujeto cognitivo, asunto importante que hay que tener en cuenta, porque el constructivismo surge como oposición a concepciones conductistas e innatistas, cuya premisa básica es que el sujeto cognitivo es inexistente, mientras lo que pretende defender el constructivismo es que, en realidad, el sujeto es un "constructor" activo de sus estructuras de conocimiento.

3. El "rescate" del sujeto cognitivo nos remite a la cuestión de la relación especial que existe entre sujeto y estímulo en toda posición constructivista. En las posiciones epistemológicas más clásicas dentro de la psicología, la relación entre el sujeto y el estímulo es absolutamente reactiva. O sea, el sujeto, más que un "sujeto", es un mero receptáculo de las influencias del medio. Nos gustaría adelantar que el concepto de construcción es clave aquí para entender las distintas posiciones. Si bien es cierto que toda posición constructivista rescata el rol constructivo del sujeto, las diferencias radican en cómo se produce esa construcción dentro del aparato cognitivo.

4. Segundo: a toda posición constructivista en psicología le subyace, como piedra angular, un determinado concepto de desarrollo. Esto porque en toda posición constructivista se hace un tratamiento explícito de la evolución de un estado cognitivo a otro estado cognitivo. En suma, se trata de explicar la "construcción" de ciertas estructuras a partir de otras distintas.

5. Las diferencias observadas entre los autores constructivistas son, sin embargo, muy importantes. Por ejemplo, en Piaget el foco está en la consideración del desarrollo de estructuras psicológicas en el niño, el foco de Vigotski en la historia de la cultura y el de Maturana en la evolución de la especie humana.

6. Tercero: toda posición constructivista tiene un marcado interés por asuntos epistemológicos. A consecuencia de los dos aspectos reseñados antes, resulta ineludible aclarar preguntas tales como ¿quién conoce?, ¿cómo conoce?, ¿qué conoce? y ¿qué es conocer? Este interés resulta 
inevitable, si se tiene en cuenta que la construcción, el desarrollo y el cambio de estructuras de conocimiento son el objeto de estudio.

Adaptado de R. Rosas y C. Sebastián (2001). Piaget, Vigotski y Maturana. Constructivismo a tres voces. Buenos Aires: Aique.

Responder las siguientes consignas

1. Nombre dos figuras destacadas en cada una de las siguientes áreas:

Educación:

Filosofía:

Historia:

2. ¿Cuáles son las tres corrientes del constructivismo a las que se refiere el autor?

3. Sintetice las características fundamentales del constructivismo.

4. Ubique las siguientes expresiones en el texto y explique qué significan:

- sujeto cognitivo:

- rol constructivo del sujeto:

- asuntos epistemológicos:

5. ¿En qué área del conocimiento se centra el autor del texto?

6. Indique si las siguientes afirmaciones son correctas o incorrectas, según el texto.

- Los enfoques innatistas surgen en oposición a las posturas constructivistas.

correcto

incorrecto

- Según el conductismo, la relación entre el sujeto y el estímulo es reactiva.

correcto

incorrecto

- Los autores mencionados explican de manera diferente el concepto de construcción.

correcto incorrecto a) Este texto le resultó:

- muy fácil de comprender

- fácil de comprender

- normal

- difícil de comprender

- muy difícil de comprender

b) El párrafo que le resultó más fácil fue el No

Explique por qué:

c) El párrafo que le resultó más difícil fue el №

Explique por qué:

d) ¿Qué preguntas le haría a al docente para comprender mejor el texto?

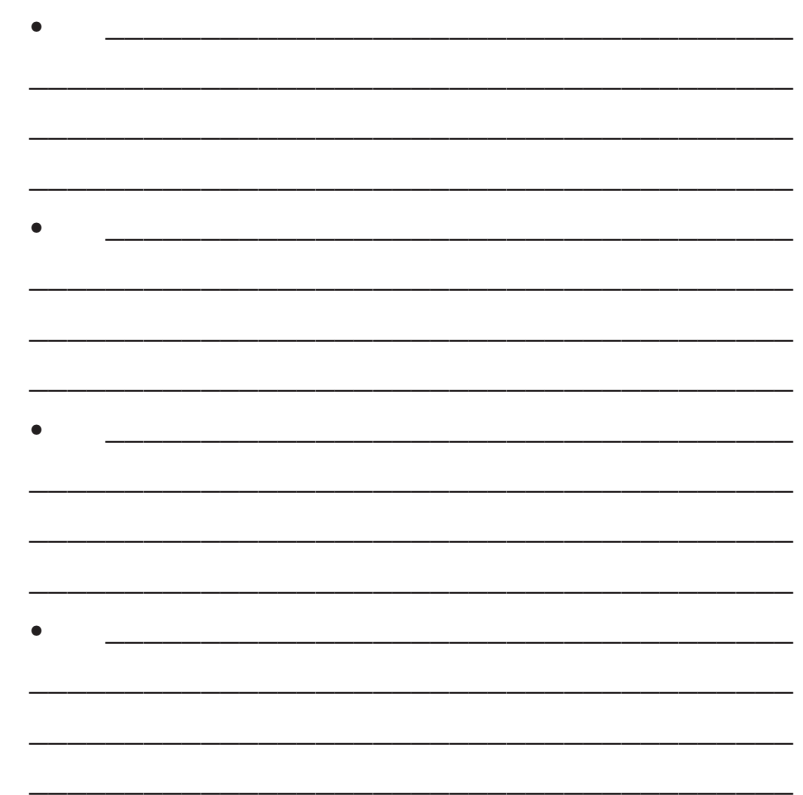




\section{Bibliografía}

Arnoux, E. (2004). "La formación docente en el área de lengua y literatura”. Conferencia plenaria de cierre del I Congreso Internacional Educación, Lenguaje, Sociedad: Tensiones educativas en América Latina. Argentina: Universidad Nacional de La Pampa.

Arnoux, E., Nogueira, S. \& Silvestre, A. (2006). Comprensión macroestructural y reformulación resuntiva de textos teóricos en estudiantes de institutos de formación de docentes primarios. Revista Signos, 39 (60), 9-30. Valparaíso, Chile: Pontificia Universidad Católica de Valparaíso.

Blanco, I. et al. (2005). Prácticas lectoras de los alumnos de los institutos de formación docente y técnica de la Provincia de Buenos Aires (República Argentina): la elección de las lecturas. Revista Actas Pedagógicas, 3(11), 98-123. Buenos Aires: Universidad de Palermo.

Ehrlich, M. (1997). Mémoire et comprehension du langage. Paris: PUF.

Freedle, R. (1997). The relevance of multiple choice reading test data in studying expository passage comprehension. Discourse Processes, Vol. 23, No 3, 399-440. Mahwah, NJ: Erlbaum.

Gombert, E. (1990). Le développement métalinguistique. Paris: PUF.

Graesser, A., Swamer S., Bagget, W \& Sell, M. (1996). New models of deep comprehension. En B. Britton \& A. Graesser (eds.). Models of understanding text. New Jersey: Lawrence Erlbaum.

Graesser, A., Olde, B., Pomeroy, V., Whitten, S., Lu, S. \& Craig, S. (2005). Inferences and questions in science text comprehension. En J. Otero \& M. Caldeira (eds.). Comprensión de los textos de ciencias. Madrid: Paidós.
Greene, L. (2005). Questioning questions. National Teaching and Learning Forum, Vol. 14, № 2 (texto electrónico).

Keyes, J. (1996). Using conceptual categories of questions to measure differences in retrieval performance. Asis, Annual Conference Proceedings (texto electrónico).

Kintsch, W. (1994). Text comprehension, memory and learning. American Psychologist, Vol. 49, No 4, 294-302. Washington: American Psychological Association.

López, G. \& Arciniegas Lagos, E. (2004). Metacognición, lectura y construcción de conocimiento. El papel de los sujetos en el aprendizaje significativo. Cali: Universidad del Valle, Unesco.

Martínez Solís, M.C. et al. (2004). Discurso y aprendizaje. Cali: Universidad del Valle, Unesco.

Mateos, M. (2001). Metacognición y educación. Buenos Aires: Aique.

Palincsar, A. \& Brown, A. (1984). Reciprocal teaching of comprehension-monitoring activities. Cognition and instruction, 1, 117-175. New Jersey: Lawrence Erlbaum.

Parodi S., G. (2005). Comprensión de textos escritos. Buenos Aires: Eudeba, Col. Enciclopedia Semiológica.

Peronard, M., Crespo, N. \& Velásquez, I. (2000). La evaluación del conocimiento metacomprensivo en alumnos de educación básica. Revista Signos, 33(47), 167-180. Valparaíso, Chile: Pontificia Universidad Católica de Valparaíso.

Otero, J. \& Graesser, A. (2001). PREG: Elements of a model of question asking. Cognition and Instruction, Vol. 19, No 2, 143-175. New Jersey: Lawrence Erlbaum.

Silvestri, A. (en prensa). La formulación de preguntas para la comprensión de textos. Signos. 